\section{Smart design of multi- granular optical switching fabric with traffic grooming}

\author{
YiYun Wang \\ Qingji Zeng \\ Lihua Lu \\ Zhicheng Sui \\ State Key Lab of Advanced Optical Communication \\ Systems and Networks \\ Shanghai Jiao Tong University \\ Shanghai 200240, China
}

\begin{abstract}
We introduce an intelligent optical cross connect (OXC) structure based on our lambda-group model, as well as a dynamic algorithm of configuration helpful to complicated optical traffic grooming, which presents a distinctive approach of dividing granularities into specific tunnels for effective treatment. Results and figures from experiments show that the particular channel partition not only is helpful to port reduction significantly, but also improves the channel and blocking performance for dynamic connection requests. () 2006 Society of Photo-Optical Instrumentation Engineers. [DOI: 10.1117/1.2172172]
\end{abstract}

Subject terms: multigranularity; GMPLS; lambda group; OXC.

Paper 050924LR received Nov. 25, 2005; revised manuscript received Dec. 8, 2005; accepted for publication Dec. 12, 2005; published online Mar. 23, 2006.

To achieve generalized label switched paths (G-LSP) in generalized multiprotocol label switching, (GMPLS) $)^{1,2}$ which subdivide the switching types and traffic granularities, items such as lambda LSP (L-LSP) and waveband LSP (WB-LSP) have been added on multigranularity switching nodes, as discussed by many works recently. Reference 3 studies the waveband routing and wavelength assessment (WRWA) problem in hierarchical mesh networks with optical cross connects $(\mathrm{OXCs})$ that can route multiple granularity. Reference 4 presents four optical grooming OXC architectures, which imply that the connection bandwidthgranularity distribution has a large impact on network throughput and network resource efficiency. References 5-7 develop more complicated structures that can expand the total switching capacity under dynamic environment. However, for complicated mesh topology, large-capacity working fibers, and an increasing number of available channels in fiber, the current models mentioned above cannot provide powerful supports to the implementation of multinode configuration under dynamic environment with complex distribution. We carried out full research, and put forward new characteristics of multigranularity switching. The first is to direct support variable-length wavebands and the logical granularity to handle the remaining channels besides obvious local wavebands, and treat nonuniform and appropriate channels for the same routing direction as a logical group. The second is to support the granularity operation among multiple fibers and permit the combination

0091-3286/2006/\$22.00 @ 2006 SPIE and disassemble of homogeneous granularities, i.e., the virtual granularity. But virtual granularities will reduce the times of multiplexing and demultiplexing greatly in the switching node, and improve the average SNR of channels. The third is to remove some inefficient modules and layers in the multigranularity model. In this paper, we propose a new node architecture with granularity grooming based on our new lambda-group theory. Results of channel and network performance are shown.

Numerous adjacent nodes, high-capacity fibers, and complex traffic in mesh topology are basic characteristics in the multigranularity switching node. The front module of the node should be capable of confirming the scale of wavebands existing in input fibers and evaluate the potential of forming advanced granularities (such as waveband) among different fibers. We think that if a fiber has already reached a certain scale of homogeneous wavelength channels (for example 1/3 of fiber capacity) and has enough potential to form larger waveband with channels for the same routing direction from other fibers, the routing direction (label of node) is called and execute direction (ED) of this fiber. This fiber is also the execute fiber (EF) of the ED. Besides the waveband with continuous channels, the above definition also allows a special waveband in which some channels are loaded with nothing: If a waveband in a fiber has a few channels without traffic load, we define the 'waveband' as a new logical granularity, namely lambda group; If the virtual waveband (from multiple fibers) has a few channels without traffic load, we define the 'waveband' as another logical granularity, namely virtual lambda group. Also, the definition of ED and EF involve four kinds of 'waveband': normal waveband, virtual waveband, lambda group, and virtual lambda group. Note, when we recognize a virtual waveband or virtual lambda group among fibers, we have to abandon what may injure the channel performance seriously in the realization due to excessive optical treatment. There may be several EDs in a fiber. One fiber is called a nonexecute fiber (NEF) if no execute direction exists in this fiber. After the static algorithm of configuration confirms all EDs of fibers, we can estimate size and number of all advanced virtual granularities among fibers. Here the frequency range of a logical granularity or an advanced virtual granularity possible in a fiber is defined as an execute range (ER), including existed wavelength channels with the ED and channels to be filtered for the granularity. The former channels are specified as an execute area (EA), which must be within the corresponding ER; all other channels except EA channels in the fiber are channels of nonexecute area (NEA). A few wavelength channels to be filtered to expand the other fiber's waveband or lambda group are called assistant wavelength channels (AW). Figure 1(a) gives a vivid explanation of all signs. The numbers from 1 to 5 denote the routing direction or the label of neighboring node. According to our definition, ' 1 ' is the ED of fiber $x$, ' 3 ' and ' 4 ' are the ED of fiber $y$, and ' 1 ' and ' 5 ' are the ED of fiber $z$. In other words, EF of ' 1 ' is fiber $x$ and fiber $z$, EF of both ' 3 ' and ' 4 ' is fiber $y$, EF of ' 5 ' is fiber $z$, and ' 2 ' has no EF. ER and EA of every fiber are also shown in the figure. There is no Non-EF here.

Here we present a smart structure based on the Internet Engineering Task Force (IETF) models. ${ }^{1}$ All input traffic is first recognized and divided by the partition module into six 


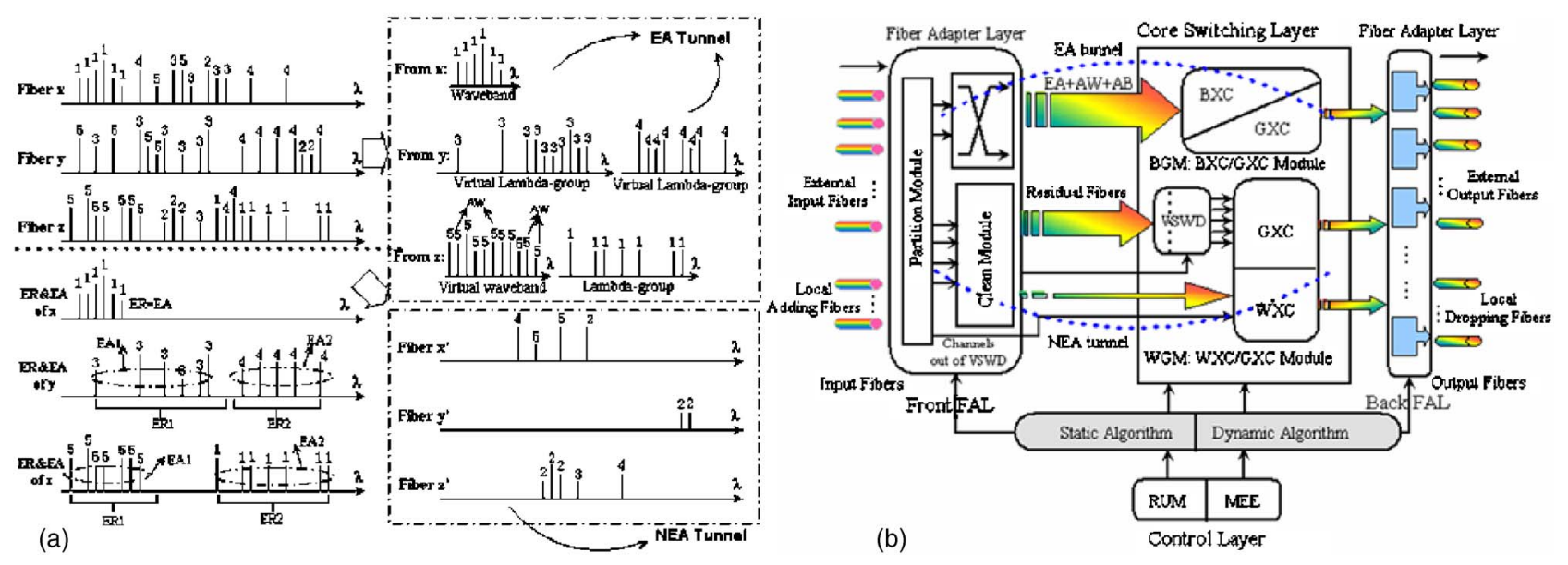

Fig. 1 (a) Example of lambda-group switching. (b) Architecture of a LGS node.

possible logical granularities to the EA tunnel and NEA tunnel. Six granularities are EA in EF (EA@EF), AW channel in EF (AW@EF), NEA within ER in EF (NEA:ER@EF), NEA out of ER in EF (NEA: $\overline{E R} @ \mathrm{EF})$, AW channel in NEF (AW@NEF) and NEA in NEF (NEA@NEF). Three clusters of granularities are large wavebands and lambda groups along the EA tunnel, complex $\lambda$ groups along the NEA tunnel, and separate wavelengths along the NEA tunnel. Three different parts in the core switching layer will be responsible for switching these granularities respectively. Since the EA tunnel can provide a higher average SNR of the channel than the SNR NEA tunnel can give, the overall performance of the channel will be improved when main traffic is processed by BGM with good policies and algorithms about traffic grooming and rearrangement of granularities as in Fig. 1(b).

Dynamic algorithm of configuration: Upon the request variation, traffic from all nodes is processed with the static algorithm BWGA. The OXC will run the dynamic module for multiple requests, i.e., DMMR as in Fig. 2(a). DMMR will assume the F-EA table $\mathrm{TA}_{2}$ figured from the dynamic $\mathrm{F}-\lambda$ table, and compare it with the F-EA table $\mathrm{TA}_{1}$ figured by BWGA at $t_{0}$. If the difference between $\mathrm{TA}_{1}$ and $\mathrm{TA}_{2}$ is large, $\mathrm{TA}_{1}$ and $\mathrm{TA}_{2}$ will be converted to the Dig-Fill form, respectively, in which each element denotes the next signal carrying on the other channel of fiber. For example, $\lambda_{31}$ in the Dig-Fill TA 1 denotes that the signal carried on the first channel of the third fiber is carrying on the first channel of the second fiber at $t_{0} . \lambda_{34}$ in the Dig-Fill TA 2 denotes that the signal carrying on the fourth channel of the third fiber is requesting to carry on the first channel of the first fiber. Then two matrices in Dig-Fill form are combined to get a ready scheme according to a relevant resource policy. Only if the ready scheme does not utilize too much resource, the node will put it into operation. Otherwise the node may look for another $\mathrm{TA}_{2}$ in view of different EAs until a preferable $\mathrm{TA}_{2}$ is confirmed. On the other hand, if the difference between $\mathrm{TA}_{1}$ and $\mathrm{TA}_{2}$ is small, the node will perform the scheme of running DMSR several times in order. DMSR is responsible for recognizing all changes of con-

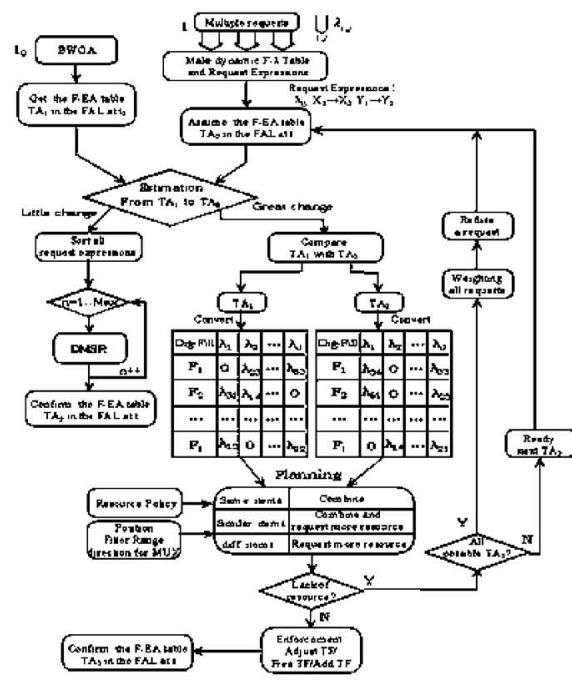

(a)

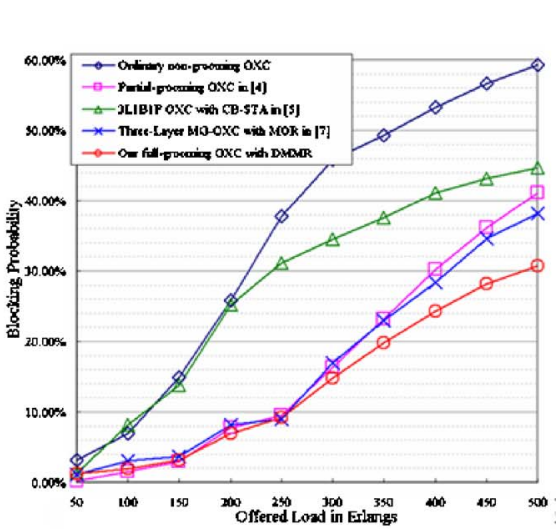

(b)

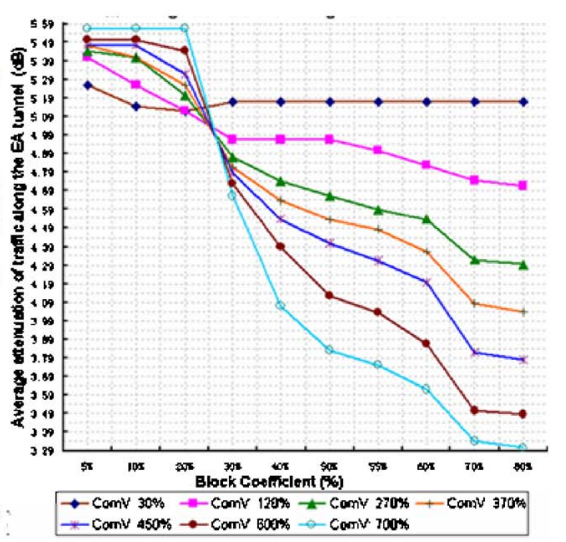

(c)

Fig. 2 (a) Flowchart of DMMR. (b) Blocking performance with increasing load. (c) Attenuation with changing $\mathrm{BC}$ along the EA tunnel. 
Table 1 Comparison of port savings.

\begin{tabular}{|c|c|c|c|}
\hline \multirow{2}{*}{ Condition } & \multirow{2}{*}{$\begin{array}{c}\text { Trad. } \\
\text { WXC }\end{array}$} & $\begin{array}{c}\text { MG-OXC } \\
\text { vs. Trad. } \\
\text { WXC }\end{array}$ & LGS OXC vs. Trad. WXC \\
\cline { 4 - 4 } & & Input $\times$ Output $(\% \times \%)$ \\
\hline $16 / F, n+5, n \backslash 1$ & $96 \times 96$ & $12.5 \%$ & $61 \times 13(36.5 \% \times 86.5 \%)$ \\
\hline $16 / F, n+5, n \backslash 2$ & $192 \times 192$ & $12.5 \%$ & $121 \times 27(37.0 \% \times 85.9 \%)$ \\
\hline $16 / F, n+5, n \backslash 3$ & $288 \times 288$ & $12.5 \%$ & $163 \times 42(43.4 \% \times 85.4 \%)$ \\
\hline $16 / F, n+5, n \backslash 4$ & $384 \times 384$ & $12.5 \%$ & $234 \times 56(39.1 \% \times 85.4 \%)$ \\
\hline $16 / F, n+5, n \backslash 5$ & $480 \times 480$ & $12.5 \%$ & $286 \times 72(40.4 \% \times 85.0 \%)$ \\
\hline $16 / F, n+1, n \backslash 2$ & $64 \times 64$ & $12.5 \%$ & $20 \times 8(68.8 \% \times 87.5 \%)$ \\
\hline $16 / F, n+2, n \backslash 2$ & $96 \times 96$ & $12.5 \%$ & $31 \times 15(67.7 \% \times 84.4 \%)$ \\
\hline $16 / F, n+3, n \backslash 2$ & $128 \times 128$ & $12.5 \%$ & $86 \times 19(32.8 \% \times 85.2 \%)$ \\
\hline $16 / F, n+4, n \backslash 2$ & $160 \times 160$ & $12.5 \%$ & $108 \times 23(32.5 \% \times 85.6 \%)$ \\
\hline $16 / F, n+5, n \backslash 2$ & $192 \times 192$ & $12.5 \%$ & $121 \times 27(37.0 \% \times 85.9 \%)$ \\
\hline $8 / F, n+5, n \backslash 3$ & $144 \times 144$ & $-25 \%$ & $94 \times 39(34.7 \% \times 72.9 \%)$ \\
\hline $16 / F, n+5, n \backslash 3$ & $288 \times 288$ & $12.5 \%$ & $163 \times 42(43.4 \% \times 85.4 \%)$ \\
\hline $24 / F, n+5, n \backslash 3$ & $432 \times 432$ & $4.2 \%$ & $250 \times 46(42.1 \% \times 89.4 \%)$ \\
\hline $32 / F, n+5, n \backslash 3$ & $576 \times 576$ & $3.1 \%$ & $350 \times 46(39.2 \% \times 92.0 \%)$ \\
\hline
\end{tabular}

WXC: wavelength cross-connect.

nections and distinguishing them, i.e., six submodules as $\lambda+@ \mathrm{EF}, \lambda-@ \mathrm{EF}, \lambda+@ \mathrm{NEF}, \lambda-@ \mathrm{NEF}, \mathrm{NA} @ \mathrm{EF}$, and NA@NEF. For instance, $\lambda+@ E F$ means adding a wavelength channel on the existing $\mathrm{EF}$ for the same $\mathrm{NH}$. $\lambda-@ N E F$ means removing a wavelength channel on the existing NEF.

ComV is the combination value and the total sum of effective sizes of possible wavebands combined with traffic from all fibers theoretically, considering all routing directions. ComV $=120 \%$ means that the ideal sum of effective sizes of possible wavebands combined reaches $120 \%$ of the capacity of a fiber. BC is the block coefficients of traffic and the mean value of the sum of effective sizes of all existing wavelength blocks from all fibers in every routing direction to the total number of fibers. For example, BC $=50 \%$ means that the mean size of all existing wavelength blocks reaches $50 \%$ of a fiber capacity.

Objective functions: The first minimizes the total cost of LGS-OXC ports. The second minimizes the average attenuation of a channel at the given offered load or near maximum offered load in every node.

Port requirement: Table 1 depicts results about port saving percentage in core layer with different switching structures. $16 / \mathrm{F}$ means 16 channels per fiber, $n+5$ means that the node $n$ is connected with five other nodes, and $n \backslash 2$ pair means that there are two pairs of unidirectional fibers between $n$ and every node connected. With different parameters, we compare cost and performance with different structures.

Channel quality and blocking performance: We simulate a dynamic network environment to evaluate the perfor- mance of different optical grooming OXCs and their corresponding grooming schemes based on 19-node NSFnet topology. Simulation focuses on the performance of overall network and some representative nodes. The network has no wavelength converter and each fiber can support 32 wavelength channels. Since recently a great amount of measurements ${ }^{2}$ confirm high-speed traffic of self-similar, we use the assumption that the connection-arrival process is self-similar and the connection-holding time follows a negative exponential distribution. We compare results of several representative fabrics from relative research ${ }^{4,5,7}$ with results of our new design in Fig. 2(b) under the same dynamic environment. With an improved dynamic algorithm, we succeed in decreasing the blocking probability below about $35 \%$ under high traffic load. We also study the average channel attenuation with changing $\mathrm{ComV}$ and $\mathrm{BC}$ at large access ratio as in Fig. 2(c). Results from simulation verify the lambda-group switching fabric has high performance and is cost-effective.

This paper first presents novel concepts such as the variable waveband, optical logical granularity, virtual waveband, and virtual lambda group, etc. Moreover, we propose a smart optical switching structure with granularity pregrooming as well as a corresponding working scheme, configuration methods, and node algorithms. The main advantages of this structure lie in the large reduction of effective fibers entering the core switching layer, a great decrease of average times of optical treatment (average quality of channels), improvement of the flexibility of multigranularity switching, and self-adjustment of resource utilization.

\section{Acknowledgments}

Financial support from National 863 Project (863-3172001AA121073) and National Natural Science Foundation of China (grant No. 69990540) are gratefully acknowledged.

\section{References}

1. R. Douville, D. Papadimitriou, L. Ciavaglia, M. Vigoureux, and E. Dotaro, "Extensions to GMPLS for waveband switching, 〈draftdouville-ccamp-gmpls-waveband-extensions-05.txt $\rangle$," work in progress (Jul. 2004).

2. C. Nuzman and J. Leuthold, "Design and implementation of wavelength-flexible network nodes," J. Lightwave Technol. 21(3), 648-663 (2003).

3. A. Kolarov, Ting Wang, B. Sengupta, and M. Cvijetic, "Impact of waveband switching on dimensioning multi-granular hybrid optical networks," Optical Network Design and Modeling Conf., pp. 371381 (2005).

4. K. Zhu and H. Zang, "A comprehensive study on next-generation optical grooming switches," IEEE J. Sel. Areas Commun. 21(7), (Sep. 2003).

5. P.-H. Ho and T. Hussein, "A novel design of optical cross-connects with multi-granularity provisioning support for the next-generation internet," ICC '03, IEEE Intl. Conf. 1(11-15), 582-587 (2003).

6. J. Leuthold and R. Ryf, "Nonblocking all-optical cross connect based on regenerative all-optical wavelength converter in a transparent demonstration over 42 nodes and $16800 \mathrm{~km}$," J. Lightwave Technol. 21(11), 2863-2870 (2003).

7. X.-J. Cao, V. Anand, Y.-Z. Xiong, and C.-M. Qiao, “A study of waveband switching with multilayer multigranular optical cross-connects," IEEE J. Sel. Areas Commun. 21(7), 1081-1095 (2003). 